\title{
ISOLATED INVOLVEMENT OF THORACOABDOMINAL AORTA TRANSITION IN TAKAYASU'S ARTERITIS MIMICKING AORTIC COARCTATION: A CASE REPORT
}

Maria de Lourdes Castro de Oliveira Figueirôa ${ }^{1, \star}$, Gustavo Luiz Behrens Pinto ${ }^{1}$, Ana Luisa Souza Pedreira ${ }^{1}$, Renata Borges de Lima ${ }^{1}$, Viviane Leal Novais ${ }^{1}$, Alisson Regis de Santana ${ }^{1}$, Mariana Oliveira Miranda ${ }^{1}$, Victor Pereira Mattos ${ }^{1}$, Kayo Cesar de Freitas Pereira ${ }^{1}$, Mittermayer Barreto Santiago ${ }^{1}$

1.Universidade Federal da Bahia, Salvador (BA), Brazil.

*Corresponding author: lourdinhafigueiroa@hotmail.com

\section{BACKGROUND}

Takayasu's arteritis (TA) is a nonatherosclerotic chronic inflammatory disease that affects the aorta and its primary branches, mainly the subclavian artery, renal artery, and common carotid artery. Involvement of other arteries usually coexists with damage to the most commonly affected arteries. The pathophysiology remains unknown, but it is associated with genetic, autoimmune, and infectious factors that cause transmural granulomatous inflammation, generating stenosis, occlusion, dilatation, and formation of aneurysms in the arteries involved. Due to its insidious evolution, sometimes asymptomatic, and the absence of specific biomarkers, sometimes it isn't easy to diagnose. Involvement of the abdominal aorta can occur in up to $50 \%$ of cases, but it is rare in isolation. The authors present a case of a young woman diagnosed with TA with isolated involvement of the thoracoabdominal aorta transition with significant stenosis, mimicking coarctation of the aorta.

\section{CASE REPORT}

A 17-year-old female presented with chest pain, palpitations, dyspnea on medium exertion, and difficult-to-control arterial hypertension for one year. Clinical examination showed a systolic murmur III/VI in the aortic focus and pulse and blood pressure asymmetry with a reduction in the lower limbs. (202/103 mmHg in the right arm, 203/111 mmHg in the left arm, $83 / 66 \mathrm{mmHg}$ in the right leg and $103 / 62 \mathrm{mmHg}$ in the left leg). The rest of the physical examination was unremarkable. Laboratory tests showed no significant changes, and inflammatory tests were negative. She underwent chest and abdominal computed tomography (CT) angiography, which showed isolated stenosis of the thoracoabdominal aorta transition with enhancement in the contrast phase suggestive of vasculitis, without evidence of aneurysmal dilatation. Based on clinical and angiographic findings, despite her atypical condition, she was diagnosed with TA (Figures 1-3). Initial treatment was instituted with prednisone $40 \mathrm{mg} /$ day and methotrexate $15 \mathrm{mg} /$ week, with partial improvement of her symptoms.

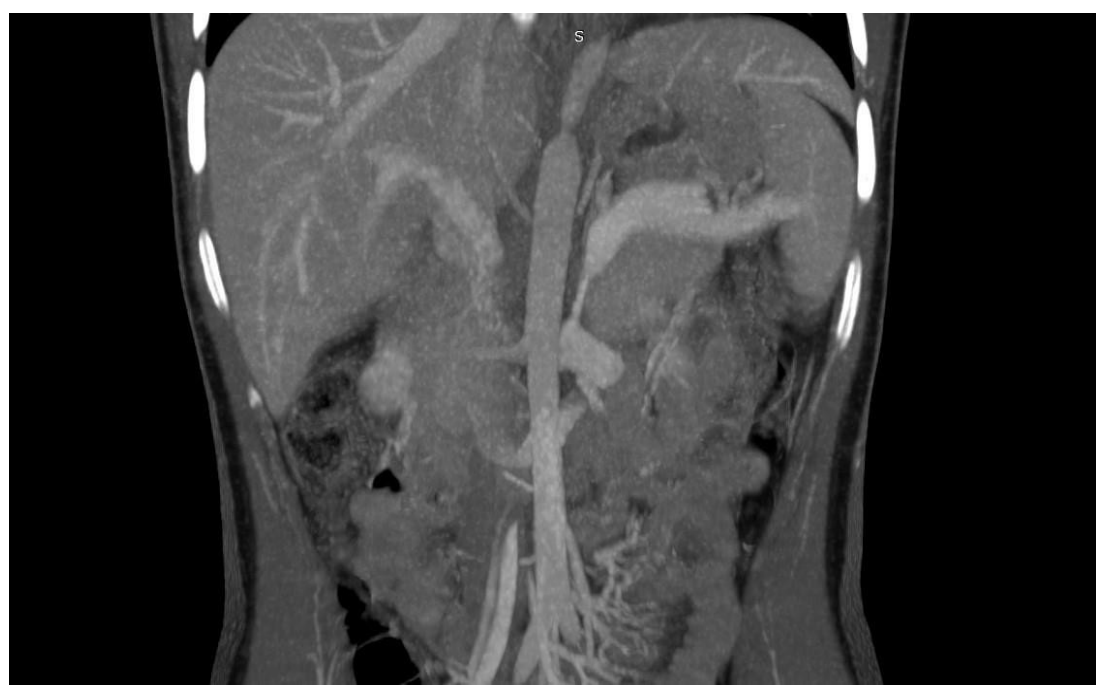

Figure 1. Chest CT angiography (coronal): significant stenosis of the thoracoabdominal transition. 


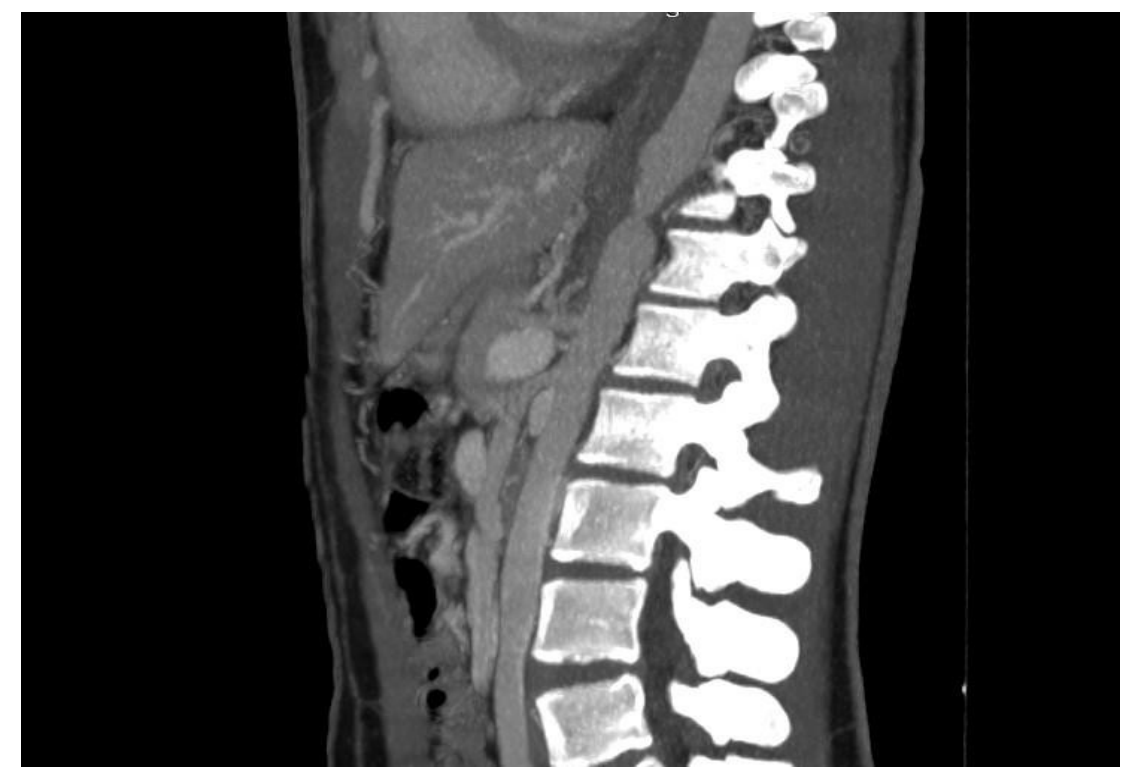

Figure 2. Chest CT angiography (sagittal): significant stenosis of the thoracoabdominal transition.

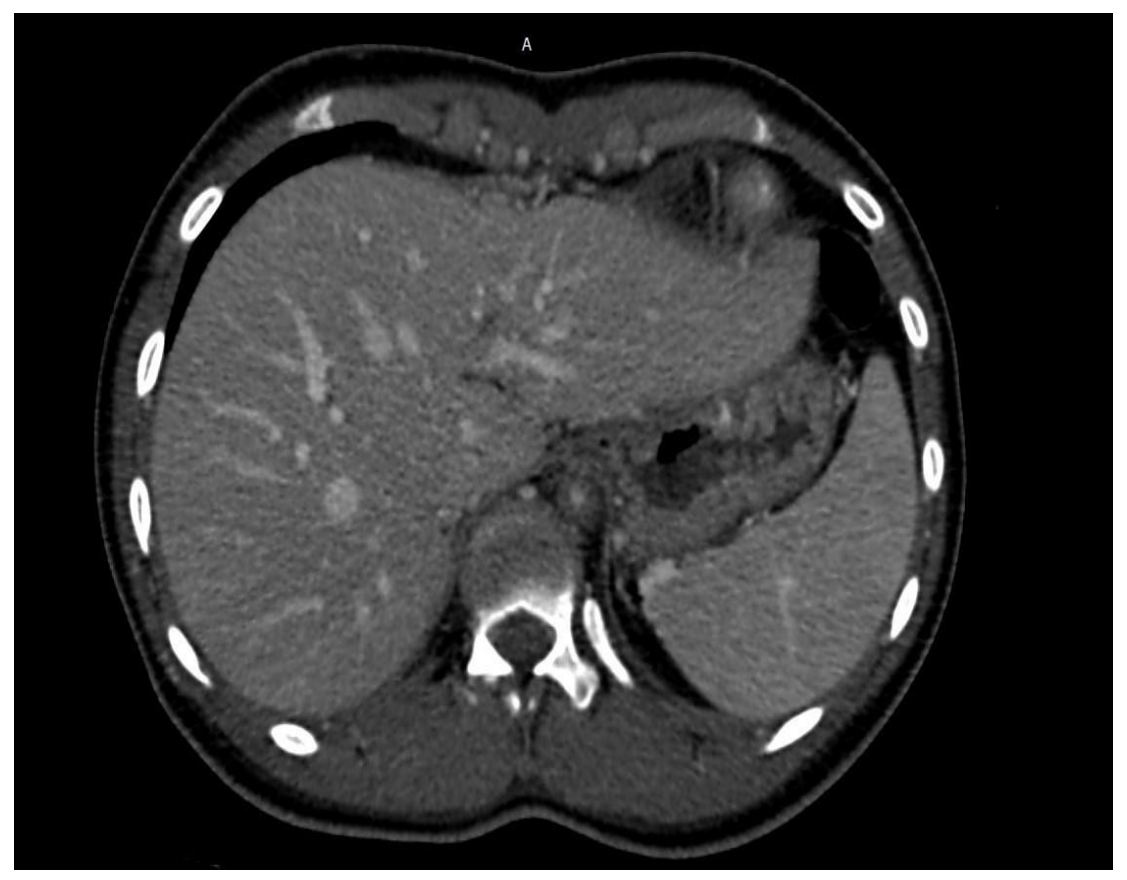

Figure 3. Chest CT angiography (axial): wall thickening of the thoracic and abdominal aorta and stenosis of the thoracoabdominal transition.

\section{CONCLUSION}

Isolated involvement of the abdominal aorta in TA is uncommon, especially when there is no evidence of aneurysmal dilatation. Atypical presentations can lead to an incorrect initial diagnosis, therapeutic delay, and unfavorable outcomes, such as severe cardiac involvement, renovascular hypertension, and cerebrovascular disease, resulting in mortality and functional incapacity.

\section{KEYWORDS}

Arteritis, Takayasu, Aortic coarctations, Aorta, Abdominal. 